BULL. AUSTRAL. MATH. SOC.

VOL. $3(1970), 289-292$.

\title{
An elementary proof of part of a classical conjecture
}

\section{R. J. Gaudet and J. L. B. Gamlen}

An elementary proof is given for the $L_{p}$ conjecture, $p>2$, which states that for a locally compact group $G, L_{p}(G)$ $(p>2)$ is closed under convolution if and only if $G$ is compact.

In this paper we present a new proof of the $L_{p}$-conjecture for $p>2$ which is elementary and much shorter than that of Rajagopalan [5].

If $G$ is a locally compact Hausdorff group with left Haar measure $m$, we define convolution as follows: If $f$ and $g$ are measurable functions for which the integral $\int_{G} f(y) g\left(y^{-1} x\right) d m(y)$ exists $a e[m]$, we say $f * g$ exists and we define $f * g(x)=\int_{G} f(y) g\left(y^{-1} x\right) d m(y)$ if this integral exists and $f * g(x)=0$ otherwise.

As usual, $L_{p}(G)(p>1)$ denotes the linear space of all measurable functions $f$ for which $\int_{G}|f|^{p} d n$ is finite. It is well known that $L_{1}(G)$ is closed under convolution in the sense that for each $f, g \in L_{1}(G)$ the convolution $f * g$ exists and is in $L_{1}(G)$. The so called " $L_{p}$-conjecture" is that $L_{p}(G)$ is closed under convolution iff $G$ is compact.

To this date, the $L_{p}$-conjecture has been proven $(p>2)$ for Received 22 May 1970. 
arbitrary groups and $(p>1)$ for solvable groups. Żelazko [7] published results in 1961 , proving the $L_{p}$-conjecture $(p>1)$ for abelian groups. In 1964, Rajagopalan and Żelazko [6] proved that if $L_{p}(G)$ is closed under convolution for some $p>1$ then the group $G$ is unimodular, and in the same paper, they proved the conjecture $(p>1)$ for solvable groups. Using his proof [4] of the $L_{p}$-conjecture $(p>2)$ for discrete groups, and a powerful reduction theorem, Rajagopalan [5] proved the $L_{p}$-conjecture $(p>2)$. In 1966 Leptin [3] introduced a two-valued function $I(G)$, and proved the $L_{p}$-conjecture among groups $G$ with $I(G)<\infty$.

It is well known (see for example Hewitt and Ross [1]) that compactness of $G$ implies that $L_{P}(G)$ is closed under convolution, for all $p \geq 1$. The following proof of the converse, for $p>2$, proceeds from first principles.

THEOREM. Let $G$ be a locally compact Hausdorff group with left Haar measure $m$. Suppose $L_{p}(G)$ is closed under convolution for some $p>2$. Then $G$ is compact.

Proof. Suppose $G$ is not compact, so that by well known arguments we may construct a sequence $\left\{a_{n}\right\} \subseteq G$ and a compact symetric neighbourhood $U$ of the identity such that $\left\{U a_{n}: n=1,2, \ldots\right\} \cup\left\{U a_{n}^{-1}: n=1,2, \ldots\right\}$ is a family of pairwise disjoint subsets of $G$. We write $\Delta$ for the modular function on $G$ :

$$
\Delta(x)=m(A x) / m(A),
$$

where $A$ is an arbitrary measurable set of positive finite measure. It is well known that $\Delta$ is independent of $A$, and that $\Delta\left(x^{-1}\right)=\Delta(x)^{-1}$. Because of the last equality we may assume without loss of generality that $\Delta\left(a_{n}\right) \geq 1$ for all $n$.

Choose a compact symetric neighbourhood $V$ of the identity such that $V^{2} \subseteq U$, and define functions $f, g$ on $G$ by: 


$$
\begin{aligned}
& f(x)=n^{-\frac{1}{2}} \Delta\left(a_{n}\right)^{-\frac{1}{p}} \text { for } x \in U a_{n}, n=1,2, \ldots ; \\
& g(x)=n^{-\frac{1}{2}} \text { for } x \in a_{n}^{-1} V, n=1,2, \ldots ;
\end{aligned}
$$

and $f, g$ vanish elsewhere.

Clearly $f, g$ are measurable, and

$$
\begin{aligned}
& \int_{G}|f|^{p} d m=\sum_{n=1}^{\infty} n^{-\frac{p}{2}} \Delta\left(a_{n}\right)^{-1} m\left(U a_{n}\right)=m(U) \sum_{n=1}^{\infty} n^{-\frac{p}{2}}<\infty, \\
& \int_{G}|g|^{p} d m=\sum_{n=1}^{\infty} n^{-\frac{p}{2}} m(V)<\infty .
\end{aligned}
$$

Thus $f, g \in L_{p}(G)$.

We now show that $f * g(t)=+\infty$ for $t \in V$. For all $t \in G$,

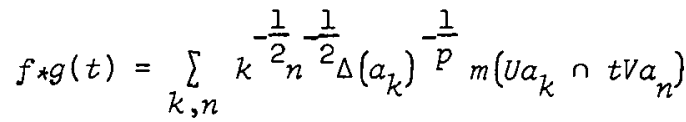

$$
\begin{aligned}
& \geq \sum_{n} n^{-1} \Delta\left(a_{n}\right)^{-\frac{1}{p}} m\left(U a_{n} \cap t V a_{n}\right) \text {. }
\end{aligned}
$$

But if $t \in V$ then $t V a_{n} \subseteq U a_{n}$, so

$$
m\left(U a_{n} \cap t V a_{n}\right)=\Delta\left(a_{n}\right) m(V) \text {. }
$$

Since $\Delta\left(a_{n}\right) \geq 1$ we deduce that

$$
f * g(t) \geq \sum_{n} n^{-1} m(V)=\infty \text { for } t \in V \text {. }
$$

This contradicts the assumption that $f * g \in L_{p}(G)$, proving the theorem. 


\section{References}

[1] Edwin Hewitt; Kenneth A. Ross, Abstract harmonic analysis. Vol. I. (Die Grundlehren der mathematischen Wissenschaften, Band 115, Academic Press, New York; Springer-Verlag, Berlin, Göttingen, Heidelberg, 1963).

[2] Horst Leptin, "Faltungen von Borelschen Massen mit ${ }_{L}^{p}$-Funktionen auf lokal kompakten Gruppen", Math. Ann. 163 (1966), 111-117.

[3] Horst Leptin, "On a certain invariant of a locally compact group", Bulz. Amer. Math. Soc. 72 (1966), 870-874.

[4] M. Rajagopalan, "On the $I^{p}$-space of a locally compact group", Colzoq. Math. 10 (1963), 49-52.

[5] M. Rajagopalan, " $L^{p}$-conjecture for locally compact groups. I", Trans. Amer. Math. Soc. 125 (1966), 216-222.

[6] M. Rajagopalan and W. Żelazko, " $L^{p}$-conjecture for solvable locally compact groups", J. Indian Math. Soc. (N.S.) .29 (1965), 87-92.

[7] W. Zelazko, "On the algebras $L_{p}$ of locally compact groups", Colzoq. Math. 8 (1961), 115-120.

[8] W. Zelazko, "A note on $L_{p}$-algebras", Colzoq. Math. 10 (1963), 53-56.

The University of Alberta,

Edmonton, Canada. 